\title{
Changes in Gaze Behavior during the Learning of the Epidural Technique with a Simulator in Anesthesia Novices
}

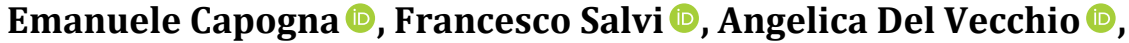 \\ Matteo Velardo (), Giorgio Capogna ${ }^{(0 *}{ }^{*}$
}

European School of Obstetric Anesthesia, EESOA Simulation Center, Roma, Italy

Email: *capogna.eesoa@gmail.com

How to cite this paper: Capogna, E., Salvi, F., Del Vecchio, A., Velardo, M. and Capogna, G. (2020) Changes in Gaze Behavior during the Learning of the Epidural Technique with a Simulator in Anesthesia Novices. Open Journal of Anesthesiology, 10, 361-370. https://doi.org/10.4236/ojanes.2020.1011032

Received: September 16, 2020

Accepted: November 6, 2020

Published: November 9, 2020

Copyright $\odot 2020$ by author(s) and Scientific Research Publishing Inc. This work is licensed under the Creative Commons Attribution International License (CC BY 4.0).

http://creativecommons.org/licenses/by/4.0/ (c) (i) Open Access

\begin{abstract}
Background: Current literature demonstrates the ability of eye tracking to provide reliable quantitative data as an objective assessment tool, with potential applications to medical and surgical training to improve performance. Objective: The aim of this study was to evaluate the changes in gaze behavior in anesthesia novice trainees when performing a simulated epidural technique before and after a hands-on training on the epidural simulator. Methods: We enrolled 48 novice trainees who had never previously performed an epidural block. After a standardized learning module, each trainee practiced the epidural procedure on the epidural simulator while wearing a pair of eye tracking glasses (Tobii Pro Glasses $50 \mathrm{~Hz}$ wearable wireless eye tracker). After this baseline recording, each trainee spent two hours practicing with the epidural simulator and afterwards once again performed the eye tracking epidural procedure. Eye tracking metrics and epidural learning (duration of the procedure and number of attempts) before and after the simulated practice were recorded. Results: The duration of the epidural procedure and of the epidural needle advancement phase $(\mathrm{P}<0.05)$ and the number of epidural attempts $(\mathrm{P}<0.001)$ were reduced after the tutorial. When considering the eye tracking metrics, after the tutorial, the number of visit counts decreased and their duration increased $(\mathrm{P}<0.05)$. The number of epidural needle insertions (additional attempts) showed a significant positive correlation with the visits number $(\mathrm{aOR}=2.02(95 \% \mathrm{CI}=1.26-3.55 ; \mathrm{P}=0.008))$ and a significant negative correlation with the visit duration $(\mathrm{aOR}=0.65(95 \% \mathrm{CI}=0.39-0.99$; $\mathrm{P}=0.05)$ ). Conclusion: We observed significant changes in gaze behavior associated with increased performance during the epidural technique learning with a simulator in anesthesia trainees who had never previously performed an epidural block. These results may create a prototype for future studies on
\end{abstract}


eye tracking technique as a teaching and evaluating tool in simulation.

\section{Keywords}

Simulation, Epidural Technique, Eye Tracking

\section{Introduction}

Epidural block is a complex procedure and requires cognitive skills such as the knowledge of the anatomy along with psychomotor skills such as the skills required to perform the technique. The learning curve is one of the most common tools to assess how the physician in training is progressing at a skill, but the achievement of competence is highly dependent on the subjective and qualitative nature of the definition of learning.

Eye tracking is the process of measuring an individual's eye movements, using a device called an eye-tracker, to register his attentional behavior while performing a task.

The principle underlying the use of eye-tracking technology is the "eye-mind" theory that suggests there is a relationship between where an individual looks (fixates) and the insight into the cognitive processes that guide this scanning, essentially what he is paying attention to, or thinking about at that point in time.

Although cognitive processes are complex, and it is possible that an individual may be simultaneously fixating on one thing, thinking about something else, studies have demonstrated that an individual's attention and thoughts at a given point in time most likely correspond to their point of fixation [1] [2].

In the medical field, perception and visual expertise have a high impact on work efficiency and effectiveness, as well as on the correctness of analysis and diagnosis.

Eye tracking has been successfully used for quantitative proficiency assessment and may be a new promising tool to evaluate competency. Current literature demonstrates the ability of eye tracking to provide reliable quantitative data as an objective assessment tool, with potential applications to medical and surgical training to improve performance [3].

Eye metrics can consistently distinguish non-experts from experts, and many studies report that as proficiency in a task improves, there is an increase in focused attention and visual representation during its performance [4] [5] [6] [7] [8].

Using the eye-tracking methodology in medical students, Zumwalt et al. [9] demonstrated that education may quite quickly affect visual behavior as new knowledge is gained. Soh et al. [10] examined trainee radiologist readers while they attempted to detect breast lesions on radiologic images before and after an e-learning tutorial, observing an improvement in lesion detection as demonstrated by improved eye-tracking metrics.

We performed this study to evaluate the changes in gaze behavior in anesthe- 
sia novice trainees when performing a simulated epidural technique before and after a hands-on training using an epidural simulator.

\section{Methods}

Forty-eight anesthesia trainees volunteered from the University of Catania Medical School to be enrolled in this prospective, observational study. Each participant gave written informed consent and privacy, confidentiality and anonymity were fully guaranteed by the EESOA Research Board.

In our Region simulation centers do not have access to a formal ethical approval process. Our study was eligible for Ethical Committee's exemption, in accordance with US Federal Human Subject Regulations-Protection of Human Subjects, due to the nature of the study itself, as no patients were involved, the trainees participating were volunteers, researchers ensured that those taking part in the research would not be caused distress, all participants' personal and other data were completely anonymized, and all the investigators had no conflict of interest and were not involved in any of the participants' university teaching programs. In addition, the study was in line with the Healthcare Simulationist Code of Ethics supported by the Society for Simulation in Healthcare [11].

All the novice trainees included in this study had never previously performed an epidural block.

All trainees received a standardized three-hour learning module on epidural anatomy and technique as described elsewhere [12] and subsequently practiced the epidural procedure on the epidural simulator while wearing a pair of eye tracking glasses. Each trainee then spent two hours practicing the epidural technique with the epidural simulator, under the supervision of a trained instructor. At the end of this individual hands-on tutorial, every trainee was asked to perform once again the epidural procedure, wearing the eye tracking glasses.

For this study we used a commercially available Tobii Pro Glasses $50 \mathrm{~Hz}$ wearable wireless eye tracker. This system can measure eye movements using cameras integrated into the eyeglasses which record the corneal reflection of infrared lighting to track pupil position, mapping the subject's focus of attention on video recordings of the subject's field of vision (gaze). In addition to tracking gaze, this system enables the measurement of various eye metrics including fixation frequency and dwell time, used as a surrogate measure of perceived stimulus importance [13].

All the eye-tracked epidural procedures were recorded immediately after accurate individual calibration, during which the participant, after wearing the glasses unit, focused on the center of the calibration target.

All the eye tracking video-recordings were stored and analyzed by using a Tobii Pro Lab Software. We selected five areas of interest (AOI), to define regions of a displayed stimulus, and to extract metrics specifically for those regions. The areas were the following: 1) point of the epidural needle at its insertion into the skin; 2) shaft of the epidural needle; 2) hub of the needle; 3 ) barrel 
of the syringe; 4) plunger of the syringe; 5) other fields of view.

We also divided the epidural procedure into three phases: 1) epidural needle insertion and advancement; 2 ) loss of resistance procedure (LOR); 3) duration of the whole epidural simulated procedure.

Number and duration of fixations, visit counts and their duration for each area of interest and for each phase before and after the hands-on tutorial, were examined.

Fixations are represented as discrete samples of almost stable points where the eye is looking. Numbers of visits are defined as number of visits that occur during an interval of time, and specific to a target AOI. Visit duration is the elapsed time between the start of the first fixation on the AOI until the end of the last fixation on the AOI.

Eye tracking metrics were mapped as gaze plots and heat maps. Heat maps and gaze plots are data visualizations that can communicate important aspects of visual behavior clearly and with great power. Gaze plots show the location, order, and time spent looking at locations on the stimulus. Time spent looking, most commonly expressed as fixation duration, is shown by the diameter of the fixation circles. The longer the look, the larger the circle.

Heat maps show how looking is distributed over the stimulus and can effectively reveal the focus of visual attention.

For the purpose of the study we used a validated, modified version of a commercially available epidural simulator (P61, 3B Scientific) which can realistically reproduce the anatomical features of the layers the needle must pass through to reach the epidural space [14].

Number of attempts and total duration of the epidural procedure before and after the hands-on tutorial were recorded. An attempt was defined as a complete withdrawal of the needle from the skin and its reinsertion at the same or at a different interspace. The total duration of the procedure was defined as the length of time between the insertion of the epidural needle into the skin of the simulator and the completion of the loss of resistance technique (finding of the epidural space).

\section{Statistical Analysis}

The power analysis was set according to a parametric model (paired t-test) in order to set $90 \%$ test power and a $95 \%$ significance level and resulted in a required sample size of 44 observations. A paired t-test was used to evaluate the differences between the variables observed before and after the hands-on tutorial.

A logistic regression model was used to evaluate the correlation between the dependent variable "number of epidural needle insertions" and the control variable "eye tracking metrics". It was not possible to make a priori power analysis because the impossibility to make assumptions on number of insertions before the experiment so a post hoc power analysis was carried out for a logistic regression model. Considering the enrolled sample size and setting the significance 
level of $95 \%$ and the effect size of 0.5 , the power of test for the logistic regression model resulted to be $80 \%$. The adjusted odds ratio (aOR) was calculated from the logistic regression parameters.

\section{Results}

All the trainees completed the task. Two eye tracking recordings were excluded for technical reasons, leaving 46 for the analysis.

The duration of the epidural procedure and of the epidural needle advancement phase $(P<0.05)$ and the number of epidural attempts $(P<0.001)$ were reduced after the tutorial (Table 1 ).

When considering the eye tracking metrics, no differences in the number and duration of fixations were noted. After the tutorial, the number of visit counts decreased and their duration increased $(\mathrm{P}<0.05)$ (Table 2). No differences were noted between the groups during the LOR procedure phase.

The number of epidural needle insertions (additional attempts) showed a significant positive correlation with the visits number $(\mathrm{aOR}=2.02(95 \% \mathrm{CI}=1.26$ 3.55; $\mathrm{P}=0.008)$ ) and a significant negative correlation with the visit duration $(\mathrm{aOR}=0.65(95 \% \mathrm{CI}=0.39-0.99 ; \mathrm{P}<0.05))$.

The radar chart of mean visit count and mean visit duration (seconds) in the assessed AOI during the epidural procedure is reported in Figure 1.

Examples of typical gaze plot and heat map before and after the tutorial are shown in Figure 2.

Table 1. Duration of the epidural procedure and number of additional attempts before and after the practical tutorial.

\begin{tabular}{ccccccccc}
\hline & \multicolumn{3}{c}{ Before tutorial } & \multicolumn{3}{c}{ After tutorial } & t-test \\
\cline { 2 - 6 } & mean & $95 \%$ CI & SD & mean & $95 \%$ CI & SD & \\
\hline $\begin{array}{c}\text { Duration of the epidural } \\
\text { needle advancement phase } \\
\text { (seconds) }\end{array}$ & 22.6 & $(16.8-27.6)$ & 18.5 & 17.4 & $(14.6-20.1)$ & 9.4 & $\mathrm{P}<0.05$ \\
$\begin{array}{c}\text { Total duration of epidural } \\
\text { procedure (seconds) }\end{array}$ & 252.0 & $(196.3-308.6)$ & 193.4 & 178.5 & $(116.2-240.8)$ & 212.2 & $\mathrm{P}<0.05$ \\
$\begin{array}{c}\text { Number of additional } \\
\text { epidural attempts (count) }\end{array}$ & 2 & $(1-4)$ & 1.5 & 0 & $(0-1)$ & 1.1 & $\mathrm{P}<0.001$ \\
\hline
\end{tabular}

Table 2. Eye tracking metrics, observed during the epidural needle advancement phase, before and after the practical tutorial.

\begin{tabular}{ccccccccc}
\hline & \multicolumn{3}{c}{ Before tutorial } & \multicolumn{3}{c}{ After tutorial } & t-test \\
\cline { 2 - 7 } & mean & $95 \% \mathrm{CI}$ & SD & mean & $95 \% \mathrm{CI}$ & SD & \\
\hline Total number of fixations (count) & 17 & $(12-20)$ & 13.1 & 14 & $(11-17)$ & 9.4 & \\
Duration of fixations (seconds) & 0.9 & $(0.8-1)$ & 0.5 & 1.0 & $(0.8-1.2)$ & 0.6 & \\
Visit count (count) & 3 & $(2.2-3.1)$ & 1.1 & 2 & $(1.8-2.5)$ & 1.3 & $\mathrm{P}<0.05$ \\
Visit duration (seconds) & 1.4 & $(1.2-1.6)$ & 0.7 & 1.8 & $(1.4-2.1)$ & 1.2 & $\mathrm{P}<0.05$ \\
\hline
\end{tabular}




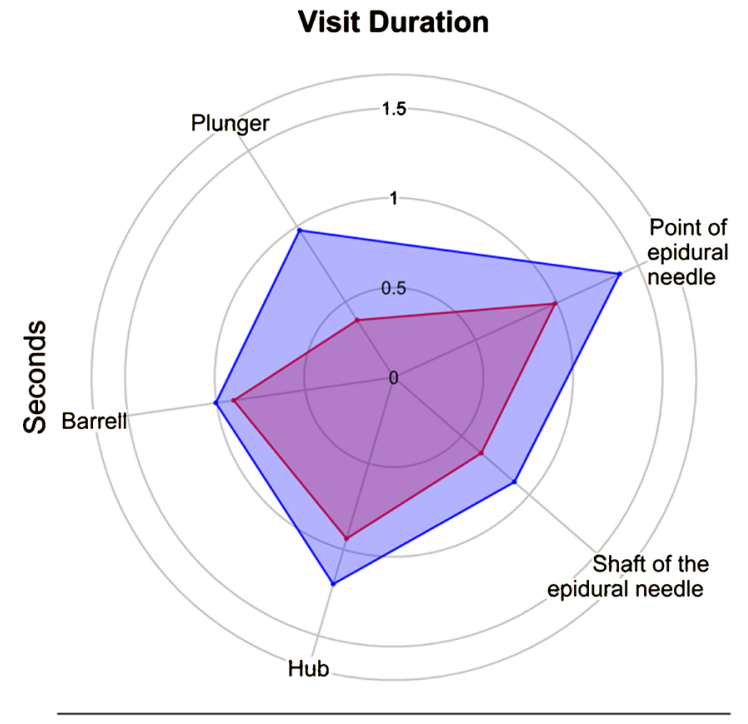

- Pre Post

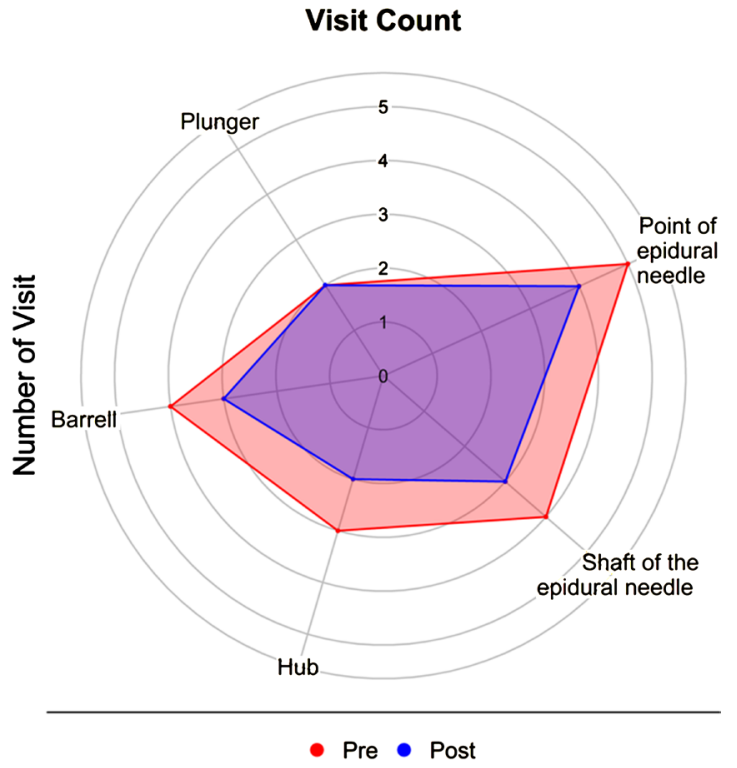

- Pre - Post

Figure 1. Radar chart of mean visit count and mean visit duration (seconds) in the assessed AOI during the epidural procedure. Pre: before the practical tutorial with simulation; Post: after the tutorial.
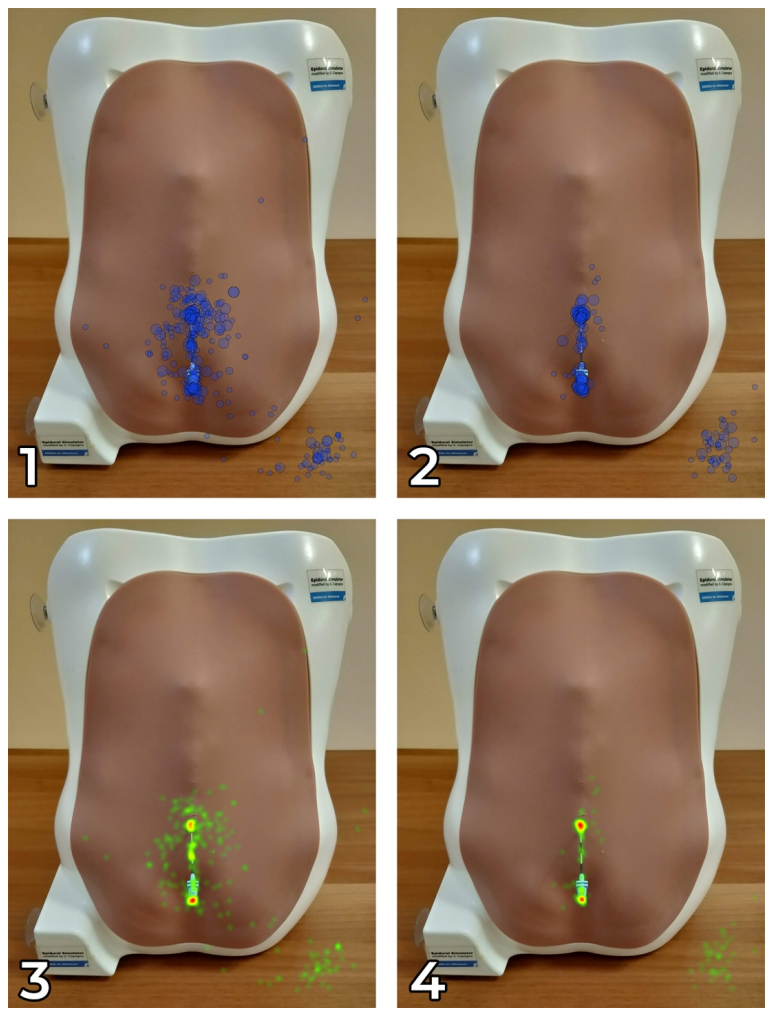

Figure 2. Gaze plots (above) and heat maps (below) before ((1), (3)) and after ((2), (4)) the tutorial. The photos show the representative example of changes in eye tracking metrics in the case of the trainee who improved after the practical tutorial.

\section{Discussion}

In this study we have examined changes in gaze behavior during the initial training with an epidural simulator in novice anesthesia trainees who had never 
previously performed an epidural block.

The assessment of gaze and attention and their relationship to expertise is the most common use of eye tracking [3]. Significantly greater fixation of relevant anatomic targets in laparoscopic procedures was demonstrated by expert surgeons and similar findings were reported for studies in other non-surgical specialties [4] [5] [6] [7] [8]. Scan path analysis has shown that novices had a much wider search strategy, compared to their more experienced colleagues [4].

In one preliminary study on epidural simulator[15], expert anesthesiologists had an overall shorter duration of fixations during the epidural procedure, and spent more time fixating a more specific target location such as the point of the epidural needle rather than the syringe's barrel, whilst trainees split their attention between tracking their tools and the target location.

Interestingly, in this study we have observed significant changes in visit counts and duration, but not in number and duration of fixations, which are the eye tracking metrics associated to experience and expert behavior [4] [5] [6] [7] [8].

A visit is the period of time when a participant first focuses on a region until the person looks away from that region and may consist of at least one fixation but could include dozens depending on the size and content in the region. Visit count and total visit duration can be informative when examining individual participant's interest or ease of understanding.

Our study has evidenced more visit counts of shorter duration before the practical tutorial and less visit counts of longer duration after it, which may suggest the acquisition of an initial competence and a more focused attention after the tutorial.

These eye tracking pattern changes might represent the early stages of a rudimentary transition from novice to a more capable (but not still expert) behavior.

In addition, we noted that changes in visit count and duration were associated with increased performance in carrying out the epidural practical task (number of attempts), and therefore it could be assumed that visit patterns may reflect the very initial process of learning, whereas the fixation patterns may mirror the proficient behavior.

The changes we have observed may reflect the perceptual learning (the improvement of perceptual abilities with practice) which, in turn, reflects plasticity in the adult visual system [16]. We hypothesize that exposure to the tutorial might have triggered some learning-related neural changes at an early level of visual processing, representing stimuli relating to task performance [17]. The results of our study are not only purely speculative, but pave the way for future application developments of teaching with simulator task trainers.

The changes in eye tracking metrics correlated well with the number of epidural attempts which may suggest the adequacy of eye tracking as a tool to evaluate the advancements of the trainee's learning.

In anesthesia, a huge amount of information is presented visually. However, little is known about the anesthetists' distribution of visual attention to gather 
the relevant information to correctly perform a practical technique. The attention of the operator selects the relevant sources of information from the sensory input and determines which information will be perceived and processed to accomplish the skill, in our case, the epidural technique. This process is performed by the working memory that takes into account the knowledge, expectations and mental models of the epidural anatomy and technique.

Eye-tracking provides an informative metric and may be used to develop an objective assessment tool, with which trainees may be objectively monitored during their training period.

Using eye tracking to help understand the learning progress of the trainee when performing a practical activity could also supplement the methodology of utilizing gaze behavior to aid in teaching. Chetwood et al. [18] have shown that using eye-tracking technology to provide visual instruction improves completion times and reduces errors in a simulated laparoscopic surgery environment. Although this reported technology requires significant development, the future potential applications are wide-ranging.

\section{Conclusions}

In this study we were able to observe subtle, but significant, changes in gaze behavior associated with increased performance during learning of epidural technique with a simulator in anesthesia novices who had never previously performed an epidural block.

We believe that our paper may create a paradigm for future studies on the evolution of eye tracking techniques as a teaching tool and to estimate the progress of learning.

\section{Acknowledgments}

We thank Lorena Delvino, Marketing Analysis Solutions Manager SrLabs, Milano, Andrea Di Giacinto, Chief Technology Officer SrLabs, Milano, for their assistance with the eye tracking recording system and Prof. Marinella Astuto, MD, Head Department of Anesthesiology, University of Catania, for her kind assistance with the volunteer trainees.

\section{Funding}

This research did not receive any specific grant from funding agencies in the public, commercial, or not-for-profit sectors.

\section{Data Availability}

The data used to support the findings of this study are available from the corresponding author upon request.

\section{Conflicts of Interest}

The authors declare no conflicts of interest regarding the publication of this paper. 


\section{References}

[1] Just, M.A. and Carpenter, P.A. (1980) A Theory of Reading: From Eye Fixations to Comprehension. Psychological Review, 87, 329-354.

https://doi.org/10.1037/0033-295X.87.4.329

[2] Deubel, H., O’Regan, J.K. and Radach, R. (2000) Commentary on Section 2-Attention, Information Processing, and Eye Movement Control. In: Kennedy, A., Radach, R., Heller, D. and Pynte, J., Eds., Reading as a Perceptual Process, Elsevier, Oxford, 355-374. https://doi.org/10.1016/B978-008043642-5/50017-6

[3] Tien, T., Pucher, H., Sodergren, M.H., Kumuthan, S., Yang, G.Z. and Darzi, A. (2014) Eye Tracking for Skills Assessment and Training: A Systematic Review. Journal of Surgical Research, 191, 169-178. https://doi.org/10.1016/j.jss.2014.04.032

[4] Wood, G., Knapp, K.M., Rock, B., Cousens, C., Roobottom, C. and Wilson, M.R. (2013) Visual Expertise in Detecting and Diagnosing Skeletal Fractures. Skeletal Radiology, 42, 165-172. https://doi.org/10.1007/s00256-012-1503-5

[5] Wilson, M.R., Vine, S.J., Bright, E., Masters, R.S., Defriend, D. and McGrath, J.S. (2011) Gaze Training Enhances Laparoscopic Technical Skill Acquisition and Multi-Tasking Performance: A Randomized, Controlled Study. Surgical Endoscopy, 25, 3731-3739. https://doi.org/10.1007/s00464-011-1802-2

[6] Tien, T., Pucher, P.H., Sodergren, M.H., Sriskandarajah, K., Yang, G.Z. and Darzi, A. (2015) Differences in Gaze Behaviour of Expert and Junior Surgeons Performing Open Inguinal Hernia Repair. Surgical Endoscopy, 29, 405-413. https://doi.org/10.1007/s00464-014-3683-7

[7] Wilson, M., McGrath, J., Vine, S., Brewer, J., Defriend, D. and Masters, R. (2010) Psychomotor Control in a Virtual Laparoscopic Surgery Training Environment: Gaze Control Parameters Differentiate Novices from Experts. Surgical Endoscopy, 24, 2458-2464. https://doi.org/10.1007/s00464-010-0986-1

[8] Richstone, L., Schwartz, M.J., Seideman, C., Cadeddu, J., Marshall, S. and Kavoussi, L.R. (2010) Eye Metrics as an Objective Assessment of Surgical Skill. Annals of Surgery, 252, 177-182. https://doi.org/10.1097/SLA.0b013e3181e464fb

[9] Zumwalt, A.C., Iyer, A., Ghebremichael, A., Frustace, B.S. and Flannery, S. (2015) Gaze Patterns of Gross Anatomy Students Change with Classroom Learning. Anatomical Sciences Education, 8, 230-241.

[10] Soh, B.P., Reed, W.M., Poulos, A. and Brennan, P.C. (2013) E-Tutorial Improves Students' Ability to Detect Lesions. Radiologic Technology, 85, 17-26.

[11] Society for Simulation in Healthcare (2018) Healthcare Simulationist Code of Ethics. https://www.ssih.org/SSH-Resources/Code-of-Ethics

[12] Capogna, G. (2020) Teaching the Epidural Block. In: Capogna, G., Ed., Epidural Technique in Obstetric Anesthesia, Springer Nature, Switzerland, 154-156. https://doi.org/10.1007/978-3-030-45332-9 10

[13] Duchowski, A.T. (2007) Eye Tracking Methodology: Theory and Practice. Springer, New York.

[14] Capogna, G., Coccoluto, A., Capogna, E. and Del Vecchio, A. (2018) Objective Evaluation of a New Epidural Simulator by the CompuFlo ${ }^{\oplus}$ Epidural Instrument. Anesthesiology Research and Practice, 2018, Article ID: 4710263. https://doi.org/10.1155/2018/4710263

[15] Capogna, E., Salvi, F., Delvino, L., Di Giacinto, A. and Velardo M. (2020) Novice and Expert Anesthesiologists' Eye-Tracking Metrics during Simulated Epidural Block: A Preliminary, Brief Observational Report. Local and Regional Anesthesia, 
13, 105-109. https://doi.org/10.2147/LRA.S267879

[16] Szpiro, S.F., Spering, M. and Carrasco, M. (2014) Perceptual Learning Modifies Untrained Pursuit Eye Movements. Journal of Vision, 14, 8.

https://doi.org/10.1167/14.8.8

[17] Karni, A. (1996) The Acquisition of Perceptual and Motor Skills: A Memory System in the Adult Human Cortex. Brain Research. Cognitive Brain Research, 5, 39-48. https://doi.org/10.1016/S0926-6410(96)00039-0

[18] Chetwood, A.S., Kwok, K.W., Sun, L.W., Mylonas, G.P., Clark, J., Darzi, A. and Yang, G.Z. (2012) Collaborative Eye Tracking: A Potential Training Tool in Laparoscopic Surgery. Surgical Endoscopy, 26, 2003-2009.

https://doi.org/10.1007/s00464-011-2143-x 